\title{
Synchronization of Chaotic Delayed Neural Networks via Impulsive Control
}

\author{
Yang Fang, ${ }^{1}$ Kang Yan, ${ }^{1}$ and Kelin $\mathrm{Li}^{2}$ \\ ${ }^{1}$ School of Automation and Electronic Information, Sichuan University of Science \& Engineering, Sichuan 643000, China \\ ${ }^{2}$ Institute of Nonlinear Science and Engineering Computing, Sichuan University of Science \& Engineering, Sichuan 643000, China \\ Correspondence should be addressed to Kelin Li; lkl@suse.edu.cn
}

Received 12 August 2013; Accepted 11 February 2014; Published 23 March 2014

Academic Editor: Han H. Choi

Copyright (c) 2014 Yang Fang et al. This is an open access article distributed under the Creative Commons Attribution License, which permits unrestricted use, distribution, and reproduction in any medium, provided the original work is properly cited.

\begin{abstract}
This paper is concerned with the impulsive synchronization problem of chaotic delayed neural networks. By employing Lyapunov stability theorem, impulsive control theory and linear matrix inequality (LMI) technique, several new sufficient conditions ensuring the asymptotically synchronization for coupled chaotic delayed neural networks are derived. Based on these new sufficient conditions, an impulsive controller is designed. Moreover, the stable impulsive interval of synchronized neural networks is objectively estimated by combining the MATLAB LMI toolbox and one of the two given equations. Two examples with numerical simulations are given to illustrate the effectiveness of the proposed method.
\end{abstract}

\section{Introduction}

During implementation of artificial neural networks, time delay is a very familiar phenomenon due to the finite switching speed of neurons and amplifiers. The existence of time delay possesses an important source in what cause instability and even chaotic behavior in some type of delayed neural networks (DNNs) if the parameters and time delays are appropriately chosen (see [1-6]). These kinds of chaotic neural networks have been successfully applied in chemical biology [7], combinatorial optimization [8], associative memory [9], biological simulation [10], and so on. Especially, the synchronization problem of chaotic delayed neural networks has been extensively studied over the past few decades due to its potential applications in many areas, such as secure communications [11-13], image encryption [14], image processing [15], and harmonic oscillation generation [16]. Hence, it is of great theoretical and practical significance to investigate synchronization problem of chaotic delayed neural networks.

A wide variety of schemes have been proposed for the synchronization of chaotic systems, for example, adaptive control [17], slide mode control [18], coupling control [19], feedback control [20], and impulsive control [21-25]. It is worth noting that impulsive control is characterized by the abrupt changes in the system dynamics at certain instants, which is an advantage in reducing the amount of information transmission and improving the security and robustness against disturbances especially in telecommunication network and power grid, orbital transfer of satellite. In addition, impulsive control allows the stabilization and synchronization of chaotic systems using only small control impulses. Thus, it has been widely used to synchronize chaotic neural networks (see [21, 23, 24]). In [21], Zhao and Zhang obtained some new criteria for the impulsive exponential antisynchronization of two chaotic delayed neural networks by establishing an integral delay inequality via the inequality method. $\mathrm{Li}$ et al. [23] investigated the synchronization scheme of coupled neural networks with time delays by utilizing the stability theory for impulsive functional differential equations. Zhang and Sun [24] studied the robust synchronization model of coupled delayed neural networks under general impulsive control. However, all above results do not efficiently utilize the so-called sector nonlinearity property of the activation functions of the neural networks, which leads to some conservatism of the results.

Motivated by the above discussions, the impulsive synchronization problem for chaotic delayed neural networks has not been fully investigated yet, which is still open and remains challenging. The aim of this paper is to study 
the synchronization of chaotic neural networks with timevarying delay. Some novel sufficient conditions which guarantee the coupled chaotic delayed neural networks can be asymptotically synchronized are derived based on Lyapunov stability theorem, impulsive control theory, and linear matrix inequality (LMI) technique. Moreover, the stable impulsive interval of synchronized neural networks is objectively estimated by combining the MATLAB LMI toolbox and one of the two given equations.

The organization of this paper is as follows. In Section 2, the impulsive synchronization problem is described and some necessary definitions and lemmas are given. Some new synchronization criteria are obtained in Section 3. In Section 4, two illustrative examples are given to show the effectiveness of the proposed method. Finally, conclusions are given in Section 5.

Notations. Let $\mathbb{R}$ denote the set of real numbers, let $\mathbb{R}^{+}$denote the set of positive real numbers, and let $\mathbb{R}^{n}$ and $\mathbb{R}^{n \times m}$ denote the $n$ dimensional Euclidean space and the set of all $n \times$ $m$ real matrices, respectively. $\mathbb{N}$ denotes the set of positive integers. $\|z\|$ is the Euclidean norm of the vector $z$. For any matrix $P \in \mathbb{R}^{n \times n}, P>0$ denotes that $P$ is a symmetric and positive definite matrix. If $P_{1}, P_{2}$ are symmetric matrices, then $P_{1} \leq P_{2}$ means that $P_{1}-P_{2}$ is a negative semidefinite matrix. $\lambda_{m}(P), \lambda_{M}(P)$ denote the minimum and maximum eigenvalue of matrix $P$, respectively. $P^{T}$ and $P^{-1}$ mean the transpose of $P$ and the inverse of a square matrix $P$. $I$ denotes the identity matrix with appropriate dimensions. $S_{\rho}=\{x \in$ $\left.\mathbb{R}^{n} \mid\|x\|<\rho\right\}, \mathscr{K}=\left\{\psi \in C\left(\mathbb{R}^{+}, \mathbb{R}^{+}\right) \mid \psi(t)\right.$ is strictly increasing and $\psi(0)=0\}, \mathscr{K}^{*}=\{\psi \in \mathscr{K} \mid \psi(t)<t$ for $t>0\}, \Sigma=\left\{\psi \in C\left(\mathbb{R}^{+}, \mathbb{R}^{+}\right) \mid \psi(0)=0, \psi(t)>0\right.$, for $\left.t>0\right\}$, $P C=\left\{\psi:[-\tau, 0] \rightarrow \mathbb{R}^{n}, \psi(t)\right.$ is continuous everywhere except at the finite number of points $\bar{t}$, where $\psi\left(\bar{t}^{+}\right), \psi\left(\bar{t}^{-}\right)$ exist and $\left.\psi\left(\bar{t}^{+}\right)=\psi(\bar{t})\right\}$. The notation $\star$ always denotes the symmetric block in one symmetric matrix.

\section{Problem Description and Preliminaries}

The chaotic neural networks with variable delay can be described by

$$
\begin{gathered}
\dot{x}(t)=-A x(t)+B f(x(t))+C f(x(t-\tau(t)))+J, \quad t>0, \\
x(s)=\phi(s), \quad s \in[-\tau, 0],
\end{gathered}
$$

where $x(t)=\left(x_{1}(t), x_{2}(t), \ldots, x_{n}(t)\right)^{T}$ is the neuron state vector; $A$ is a positive diagonal matrix; $B=\left(b_{i j}\right) \in \mathbb{R}^{n \times n}$ and $C=$ $\left(c_{i j}\right) \in \mathbb{R}^{n \times n}$ are the connection weight matrix and the delayed connection weight matrix, respectively; $J$ is the constant input vector; $f(x(\cdot))=\left(f_{1}\left(x_{1}(\cdot)\right), f_{2}\left(x_{2}(\cdot)\right), \ldots, f_{n}\left(x_{n}(\cdot)\right)\right)^{T}$ is the nonlinear neuron activation function which describes the behavior in which the neurons respond to each other; the time delay $\tau(t)$ is bounded as $0 \leq \tau(t) \leq \tau$, and the initial value condition $\phi(\cdot) \in P C\left([-\tau, 0], \mathbb{R}^{n}\right)$ is a piecewise right continuous function.

Throughout this paper we assume that $f(\cdot)$ satisfies assumption (H1).
(H1) (see [26]) Each function $f_{i}$ is continuous, and there exist scalars $l_{i}^{-}$and $l_{i}^{+}$such that

$$
l_{i}^{-} \leq \frac{f_{i}(a)-f_{i}(b)}{a-b} \leq l_{i}^{+}
$$

for any $a, b \in \mathbb{R}, a \neq b$, where $l_{i}^{+}$and $l_{i}^{-}$can be positive, negative, or zero.

Based on assumption ( $\mathrm{H} 1)$, we set

$$
\begin{gathered}
L_{1}=\operatorname{diag}\left(l_{1}^{+}+l_{1}^{-}, l_{2}^{+}+l_{2}^{-}, \ldots, l_{n}^{+}+l_{n}^{-}\right), \\
L_{2}=\operatorname{diag}\left(l_{1}^{+} l_{1}^{-}, l_{2}^{+} l_{2}^{-}, \ldots, l_{n}^{+} l_{n}^{-}\right) .
\end{gathered}
$$

Remark 1. In usual Lipschitz condition, it is assumed that $l_{i}^{-}=$ $-l_{i}^{+}$. Clearly, the condition (H1) is quite general and includes the usual Lipschitz conditions as a special case.

To investigate the impulsive synchronization of chaotic neural networks and consider system (1) as the drive system, the corresponding response system is given by

$$
\begin{gathered}
\dot{y}(t)=-A y(t)+B f(y(t))+C f(y(t-\tau(t)))+J, \quad t>0, \\
y(s)=\varphi(s), \quad s \in[-\tau, 0],
\end{gathered}
$$

where $y(t)=\left(y_{1}(t), y_{2}(t), \ldots, y_{n}(t)\right)^{T}$ is the neuron state vector of the response system, the initial value condition $\varphi(\cdot) \in P C\left([-\tau, 0], \mathbb{R}^{n}\right)$ is a piecewise right continuous function.

At discrete time $t_{k}$, the state variables of the drive system are transmitted to the response system as the control input such that the state vectors of the response system are suddenly changed at these instants. Therefore, the impulsive controlled response system can be written as

$$
\begin{array}{r}
\dot{y}(t)=-A y(t)+B f(y(t))+C f(y(t-\tau(t)))+J, \\
t \geq t_{0}=0, \quad t \neq t_{k}, \\
\Delta y\left(t_{k}\right)=y\left(t_{k}^{+}\right)-y\left(t_{k}^{-}\right)=W_{k}\left(y\left(t_{k}^{-}\right)-x\left(t_{k}^{-}\right)\right), \\
t=t_{k}, \quad k \in \mathbb{N}, \\
y(s)=\varphi(s), \quad s \in[-\tau, 0],
\end{array}
$$

where $\Delta y\left(t_{k}\right)$ denotes the state jumping at impulsive time instant $t=t_{k}, y\left(t_{k}^{+}\right), y\left(t_{k}^{-}\right)$and $x\left(t_{k}^{+}\right), x\left(t_{k}^{-}\right)$are the righthand and left-hand limits of the functions $y(t)$ and $x(t)$ at $t_{k}$, respectively. Moreover, $y\left(t_{k}\right)=y\left(t_{k}^{+}\right), x\left(t_{k}\right)=x\left(t_{k}^{+}\right)$. Suppose that the discrete time sequence $\left\{t_{k}\right\}$ satisfies $0<t_{1}<$ $t_{2}<\cdots$ and $\lim _{k \rightarrow \infty} t_{k}=\infty$. $W_{k}$ is the impulsive matrix.

Let $e(t)=y(t)-x(t)$ be the synchronization error, and then we can obtain the error system between (1) and (5):

$$
\begin{gathered}
\dot{e}(t)=-A e(t)+B h(e(t))+C h(e(t-\tau(t))), \\
t \geq t_{0}=0, \quad t \neq t_{k}, \\
\Delta e\left(t_{k}\right)=e\left(t_{k}^{+}\right)-e\left(t_{k}^{-}\right)=W_{k} e\left(t_{k}^{-}\right), \quad t=t_{k}, \quad k \in \mathbb{N}, \\
e(s)=\varphi(s)-\phi(s), \quad s \in[-\tau, 0],
\end{gathered}
$$

where $h(e(\cdot))=f(e(\cdot)+x(\cdot))-f(x(\cdot))$. 
The following definitions and lemmas which are useful in deriving synchronization criteria are used in the paper.

In general, the impulsive functional differential equation can be described by

$$
\begin{gathered}
\dot{x}(t)=f\left(t, x_{t}\right), \quad t \geq 0, t \neq t_{k}, \\
x\left(t_{k}^{+}\right)=J_{k}\left(x\left(t_{k}^{-}\right)\right), \quad t=t_{k}, k \in \mathbb{N}, \\
x(t)=x_{0}, \quad t \in[-\tau, 0],
\end{gathered}
$$

where $f:[0, \infty) \times P C \rightarrow \mathbb{R}^{n}$ ensures that (7) has a zero solution. $J_{k}(x): S_{\rho} \rightarrow \mathbb{R}^{n}$ for each $k \in \mathbb{N}^{+}$. For any $t \geq$ $0, x_{t} \in P C$ is defined by $x_{t}(s)=x(t+s),-\tau \leq s \leq 0$. Assume that there exists a $\rho_{1}>0\left(\rho_{1} \leq \rho\right)$ such that $x \in S\left(\rho_{1}\right)$ implies $J_{k}(x) \in S_{\rho}$ for all $k \in \mathbb{N}$.

Definition 2. The function $V(t, x):\left[t_{0}, \infty\right) \times S_{\rho} \rightarrow \mathbb{R}^{+}$is said to belong to class $\mathscr{V}_{0}$ if

(i) $V$ is continuous in each of sets $\left[t_{k-1}, t_{k}\right) \times S_{\rho}, k \in \mathbb{N}$, and for each $x \in S_{\rho}, \lim _{(t, y) \rightarrow\left(t_{k}^{-}, x\right)} V(t, y)=V\left(t_{k}^{-}, x\right)$ exists;

(ii) $V$ is locally Lipschitzian in $x \in S_{\rho}$ and for all $t \geq$ $t_{0}, V(t, 0) \equiv 0$.

Definition 3 (see [27]). For $(t, x) \in\left[t_{k-1}, t_{k}\right) \times \mathbb{R}^{n}$, the right and upper Dini's derivatives of $V \in \mathscr{V}_{0}$ are defined as

$$
D^{+} V(t, x) \equiv \lim _{h \rightarrow 0^{+}} \sup \frac{1}{h}\{V[t+h, x+h f(t, x)]-V(t, x)\} \text {. }
$$

Lemma 4 (see [28]). Assume that there exist $V \in$ $\mathscr{V}_{0}, \omega_{1}, \omega_{2} \in \mathscr{K}, \varphi \in \mathscr{K}^{*}$, and $H \in \sum$ such that

(i) $\omega_{1}(\|x\|) \leq V(t, x) \leq \omega_{2}(\|x\|)$ for $(t, x) \in\left[t_{0}, \infty\right) \times S_{\rho}$;

(ii) for all $x \in S_{\rho_{1}}, 0<\rho_{1} \leq \rho$, and $k \in \mathbb{N}, V\left(t_{k}, J_{k}(x)\right) \leq$ $\psi\left(V\left(t_{k}^{-}, x\right)\right)$;

(iii) for any solution $x(t)$ of (7), $V(t+s, x(t+s)) \leq \psi^{-1}$ $(V(t, x(t))),-\tau \leq s \leq 0$ implies that $D^{+}(V(t, x(t))) \leq$ $g(t) H(V(t, x(t)))$, where $g:\left[t_{0}, \infty\right) \rightarrow \mathbb{R}^{+}$is locally integrable, $\psi^{-1}$ is the inverse function of $\psi$;

(iv) $H$ is nondecreasing and there exist constants $\lambda_{2} \geq \lambda_{1}>$ 0 and $A>0$ such that for all $k \in N$ and $\mu>0, \lambda_{1} \leq$ $t_{k}-t_{k-1} \leq \lambda_{2}$ and $\int_{\psi(\mu)}^{\mu}(d s / H(s))-\int_{t_{k-1}}^{t_{k}} g(s) d s \geq A$;

then the zero solution of (7) is uniformly asymptotically stable.

Lemma 5 (see [29]). For any symmetric and positive definite matrix $P \in \mathbb{R}^{n \times n}$, the following inequality holds:

$$
\lambda_{m}(P) x^{T} x \leq x^{T} P x \leq \lambda_{M}(P) x^{T} x, \quad \forall x \in \mathbb{R}^{n} .
$$

Lemma 6 (see [30]). If $X, Y$ are real matrices with appropriate dimensions, then there exists a number $\varepsilon>0$ such that

$$
X^{T} Y+Y^{T} X \leq \varepsilon X^{T} X+\frac{1}{\varepsilon} Y^{T} Y .
$$

\section{Main Results}

In this section, we use the Lyapunov-like function

$$
V(t, e(t))=e^{T}(t) P e(t)
$$

to derive the asymptotically stability conditions of the zero solution of the error system (6), which implies that the drive system (1) and the response system (5) can be asymptotically synchronized.

Theorem 7. Assume that assumption (H1) holds. If there exist three $n \times n$ symmetric and positive definite matrices $P, Q_{1}, Q_{2}$, seven constants $0<d<1, \theta_{1}, \theta_{2}, \theta_{5}, \theta_{6}>0,0<\theta_{3}, \theta_{4}<2$ such that the following inequalities hold:

$$
\begin{gathered}
\Gamma \leq 0, \quad \Omega_{2}-\theta_{5} P \leq 0, \quad \Omega_{3}-d P \leq 0, \\
\ln d+\theta_{6} \sup \left\{t_{k}-t_{k-1}\right\}<0, \quad k \in \mathbb{N},
\end{gathered}
$$

where

$$
\begin{gathered}
\Gamma=\left[\begin{array}{ccc}
\Omega_{1} & P B & P C \\
\star & -\theta_{1} I & 0 \\
\star & \star & -\theta_{2} I
\end{array}\right], \quad \theta_{5} P \leq Q_{1}, \quad \theta_{6} P \geq Q_{2}, \\
\Omega_{1}=-A^{T} P-P A+\frac{\theta_{1}}{2 \theta_{3}-\theta_{3}^{2}} L_{1} L_{1}^{T}+\frac{Q_{1}}{d}-Q_{2}-\frac{2 \theta_{1}}{2-\theta_{3}} L_{2}, \\
\Omega_{2}=\frac{\theta_{2}}{2 \theta_{4}-\theta_{4}^{2}} L_{1} L_{1}^{T}-\frac{2 \theta_{2}}{2-\theta_{4}} L_{2}, \\
\Omega_{3}=\left(I+W_{k}\right)^{T} P\left(I+W_{k}\right),
\end{gathered}
$$

then the systems (1) and (5) are asymptotically synchronized.

Proof. From (11) and Lemma 5, we get

$$
\lambda_{m}(P) e^{T}(t) e(t) \leq V(t, e(t)) \leq \lambda_{M}(P) e^{T}(t) e(t) .
$$

Let $\omega_{1}(\|e(t)\|)=\lambda_{m}(P) e^{T}(t) e(t), \omega_{2}(\|e(t)\|)=\lambda_{M}(P) e^{T}(t)$ $e(t)$, and then $\omega_{1}, \omega_{2} \in \mathscr{K}$.

For all $e \in S_{\rho_{1}}, 0<\rho_{1} \leq \rho, k \in \mathbb{N}$,

$$
\begin{aligned}
V\left(t_{k}, e\left(t_{k}\right)\right)= & e^{T}\left(t_{k}\right) P e\left(t_{k}\right) \\
= & e^{T}\left(t_{k}^{-}\right)\left(I+W_{k}\right)^{T} P\left(I+W_{k}\right) e\left(t_{k}^{-}\right) \\
= & e^{T}\left(t_{k}^{-}\right)\left[\left(I+W_{k}\right)^{T} P\left(I+W_{k}\right)-d P\right] e\left(t_{k}^{-}\right) \\
& +d e^{T}\left(t_{k}^{-}\right) P e\left(t_{k}^{-}\right) \\
= & e^{T}\left(t_{k}^{-}\right)\left(\Omega_{3}-d P\right) e\left(t_{k}^{-}\right)+d V\left(t_{k}^{-}, e\left(t_{k}^{-}\right)\right) \\
\leq & d V\left(t_{k}^{-}, e\left(t_{k}^{-}\right)\right)
\end{aligned}
$$

Let $\psi(s)=d s$, and then $\psi \in \mathscr{K}^{*}$.

For any solution of (6), if

$$
V(t+s, e(t+s)) \leq \psi^{-1}(V(t, e(t))), \quad \forall s \in[-\tau, 0],
$$


that is,

$$
e^{T}(t+s) P e(t+s) \leq \frac{1}{d} e^{T}(t) P e(t), \quad \forall s \in[-\tau, 0]
$$

specially, for $s=-\tau(t)$, we have

$$
e^{T}(t-\tau(t)) P e(t-\tau(t)) \leq \frac{1}{d} e^{T}(t) P e(t) .
$$

For $t \in\left[t_{k-1}, t_{k}\right), k \in \mathbb{N}$, the right and upper Dini's derivatives of $V(t, e(t))$ along the trajectory of system (6) are obtained as follows:

$$
\begin{aligned}
& D^{+} V(t, e(t)) \\
& =e^{T}(t)\left(-A^{T} P-P A\right) e(t) \\
& \quad+\left(e^{T}(t) P B h(e(t))+h^{T}(e(t)) B^{T} P e(t)\right) \\
& \quad+\left(e^{T}(t) P C h(e(t-\tau(t)))+h^{T}(e(t-\tau(t))) C^{T} P e(t)\right) .
\end{aligned}
$$

It follows from Lemma 6 that

$$
\begin{aligned}
& e^{T}(t) P B h(e(t))+h^{T}(e(t)) B^{T} P e(t) \\
& \leq \frac{1}{\theta_{1}} e^{T}(t) P B B^{T} P e(t)+\theta_{1} h^{T}(e(t)) h(e(t)), \\
& e^{T}(t) P C h(e(t-\tau(t)))+h^{T}(e(t-\tau(t))) C^{T} P e(t) \\
& \leq \frac{1}{\theta_{2}} e^{T}(t) P C C^{T} P e(t)+\theta_{2} h^{T}(e(t-\tau(t))) h(e(t-\tau(t))) .
\end{aligned}
$$

By assumption (H1), it is easy to see that

$$
\begin{aligned}
0 & \leq \sum_{i=1}^{n}\left(l_{i}^{+} e_{i}(t)-h_{i}\left(e_{i}(t)\right)\right)\left(h_{i}\left(e_{i}(t)\right)-l_{i}^{-} e_{i}(t)\right) \\
& =e^{T}(t) L_{1} h(e(t))-h^{T}(e(t)) h(e(t))-e^{T}(t) L_{2} e(t),
\end{aligned}
$$

$$
\begin{aligned}
0 \leq \sum_{i=1}^{n}\left(l_{i}^{+} e_{i}(t-\tau(t))-h_{i}\left(e_{i}(t-\tau(t))\right)\right) \\
\quad \times\left(h_{i}\left(e_{i}(t-\tau(t))\right)-l_{i}^{-} e_{i}(t-\tau(t))\right) \\
=e^{T}(t-\tau(t)) L_{1} h(e(t-\tau(t))) \\
-h^{T}(e(t-\tau(t))) h(e(t-\tau(t))) \\
-e^{T}(t-\tau(t)) L_{2} e(t-\tau(t)) .
\end{aligned}
$$

Combining (20) and (22) together yields

$$
\begin{aligned}
e^{T}(t) & P B h(e(t))+h^{T}(e(t)) B^{T} P e(t) \\
\leq & \frac{1}{\theta_{1}} e^{T}(t) P B B^{T} P e(t) \\
& +\theta_{1}\left(e^{T}(t) L_{1} h(e(t))-e^{T}(t) L_{2} e(t)\right) \\
= & e^{T}(t)\left(\frac{1}{\theta_{1}} P B B^{T} P-\theta_{1} L_{2}\right) e(t) \\
& +\theta_{1} e^{T}(t) L_{1} h(e(t)) .
\end{aligned}
$$

From (21) and (23), we get

$$
\begin{gathered}
e^{T}(t) P C h(e(t-\tau(t)))+h^{T}(e(t-\tau(t))) C^{T} P e(t) \\
\leq \frac{1}{\theta_{2}} e^{T}(t) P C C^{T} P e(t) \\
+\theta_{2}\left(e^{T}(t-\tau(t)) L_{1} h(e(t-\tau(t)))\right. \\
\left.-e^{T}(t-\tau(t)) L_{2} e(t-\tau(t))\right) \\
=e^{T}(t)\left(\frac{1}{\theta_{2}} P C C^{T} P\right) e(t) \\
-e^{T}(t-\tau(t))\left(\theta_{2} L_{2}\right) e(t-\tau(t)) \\
+\theta_{2} e^{T}(t-\tau(t)) L_{1} h(e(t-\tau(t))) .
\end{gathered}
$$

Note that

$$
\begin{aligned}
e^{T}(t) & L_{1} h(e(t)) \\
\leq & \frac{1}{2 \theta_{3}} e^{T}(t) L_{1} L_{1}^{T} e(t)+\frac{\theta_{3}}{2} h^{T}(e(t)) h(e(t)) \\
\leq & \frac{1}{2 \theta_{3}} e^{T}(t) L_{1} L_{1}^{T} e(t) \\
+ & \frac{\theta_{3}}{2}\left(e^{T}(t) L_{1} h(e(t))-e^{T}(t) L_{2} e(t)\right), \\
e^{T}(t- & \tau(t)) L_{1} h(e(t-\tau(t))) \\
\leq & \frac{1}{2 \theta_{4}} e^{T}(t-\tau(t)) L_{1} L_{1}^{T} e(t-\tau(t)) \\
& +\frac{\theta_{4}}{2} h^{T}(e(t-\tau(t))) h(e(t-\tau(t))) \\
\leq & \frac{\theta_{4}}{2}\left(e^{T}(t-\tau(t)) L_{1} h(e(t-\tau(t)))\right. \\
& \quad+\frac{1}{2 \theta_{4}} e^{T}(t-\tau(t)) L_{1} L_{1}^{T} e(t-\tau(t)) . \\
& \\
&
\end{aligned}
$$


From (26), we can, namely, obtain

$$
\begin{aligned}
& e^{T}(t) L_{1} h(e(t)) \leq e^{T}(t)\left(\frac{1}{2 \theta_{3}-\theta_{3}^{2}} L_{1} L_{1}^{T}-\frac{\theta_{3}}{2-\theta_{3}} L_{2}\right) e(t), \\
& e^{T}(t-\tau(t)) L_{1} h(e(t-\tau(t))) \\
& \quad \leq e^{T}(t-\tau(t))\left(\frac{1}{2 \theta_{4}-\theta_{4}^{2}} L_{1} L_{1}^{T}-\frac{\theta_{4}}{2-\theta_{4}} L_{2}\right) e(t-\tau(t)) .
\end{aligned}
$$

Introducing (24), (25), (27), and (28) to (19), from condition of Theorem 7 and Schur complement [31], we obtain

$$
\begin{aligned}
& D^{+} V(t, e(t)) \\
& \leq e^{T}(t)\left(-A^{T} P-P A+\frac{1}{\theta_{1}} P B B^{T} P\right. \\
& \left.+\frac{1}{\theta_{2}} P C C^{T} P-\theta_{1} L_{2}\right) e(t) \\
& +e^{T}(t)\left(\frac{\theta_{1}}{2 \theta_{3}-\theta_{3}^{2}} L_{1} L_{1}^{T}-\frac{\theta_{1} \theta_{3}}{2-\theta_{3}} L_{2}\right) e(t) \\
& +e^{T}(t-\tau(t))\left(\frac{\theta_{2}}{2 \theta_{4}-\theta_{4}^{2}} L_{1} L_{1}^{T}-\frac{\theta_{2} \theta_{4}}{2-\theta_{4}} L_{2}\right) \\
& \times e(t-\tau(t)) \\
& -e^{T}(t-\tau(t))\left(\theta_{2} L_{2}\right) e(t-\tau(t)) \\
& =e^{T}(t)\left(-A^{T} P-P A+\frac{1}{\theta_{1}} P B B^{T} P+\frac{1}{\theta_{2}} P C C^{T} P\right. \\
& \left.+\frac{\theta_{1}}{2 \theta_{3}-\theta_{3}^{2}} L_{1} L_{1}^{T}-\frac{2 \theta_{1}}{2-\theta_{3}} L_{2}\right) e(t) \\
& +e^{T}(t-\tau(t))\left(\frac{\theta_{2}}{2 \theta_{4}-\theta_{4}^{2}} L_{1} L_{1}^{T}-\frac{2 \theta_{2}}{2-\theta_{4}} L_{2}\right) e(t-\tau(t)) \\
& \leq e^{T}(t)\left(-A^{T} P-P A+\frac{1}{\theta_{1}} P B B^{T} P\right. \\
& \left.+\frac{1}{\theta_{2}} P C C^{T} P+\frac{\theta_{1}}{2 \theta_{3}-\theta_{3}^{2}} L_{1} L_{1}^{T}-\frac{2 \theta_{1}}{2-\theta_{3}} L_{2}\right) e(t) \\
& +e^{T}(t-\tau(t))\left(\theta_{5} P\right) e(t-\tau(t)) \\
& \leq e^{T}(t)\left(-A^{T} P-P A+\frac{1}{\theta_{1}} P B B^{T} P\right. \\
& +\frac{1}{\theta_{2}} P C C^{T} P+\frac{\theta_{1}}{2 \theta_{3}-\theta_{3}^{2}} L_{1} L_{1}^{T} \\
& \left.-\frac{2 \theta_{1}}{2-\theta_{3}} L_{2}+\left(\frac{\theta_{5}}{d}-\theta_{6}\right) P\right) e(t) \\
& +\theta_{6} e^{T}(t) P e(t)
\end{aligned}
$$

$$
\begin{aligned}
& \leq e^{T}(t)\left(-A^{T} P-P A+\frac{1}{\theta_{1}} P B B^{T} P+\frac{1}{\theta_{2}} P C C^{T} P\right. \\
& \left.\quad+\frac{\theta_{1}}{2 \theta_{3}-\theta_{3}^{2}} L_{1} L_{1}^{T}-\frac{2 \theta_{1}}{2-\theta_{3}} L_{2}+\frac{Q_{1}}{d}-Q_{2}\right) e(t) \\
& \quad+\theta_{6} e^{T}(t) P e(t) \\
& \leq \theta_{6} V(t, e(t)) .
\end{aligned}
$$

Let $g(s) \equiv 1$ and $H(s)=\theta_{6} s$. Then

$$
\begin{aligned}
\int_{\varphi(\mu)}^{\mu} \frac{d s}{H(s)}-\int_{t_{k-1}}^{t_{k}} g(s) d s & =\int_{d \mu}^{\mu} \frac{d s}{\theta_{6} s}-\left(t_{k}-t_{k-1}\right) \\
& =-\frac{\ln d}{\theta_{6}}-\left(t_{k}-t_{k-1}\right) \\
& >-\frac{\ln d}{\theta_{6}}-\sup \left\{t_{k}-t_{k-1}\right\}>0 .
\end{aligned}
$$

From Lemma 4, the zero solution of system (6) is asymptotically stable. Thus, system (1) and system (5) are synchronized. The proof of Theorem 7 is completed.

Remark 8. The stable impulsive interval is associated with the impulsive matrix $W_{k}$ and the choice of parameter $\theta_{6}$. In order to reduce the man-made misleading during the prediction of stable impulsive interval, here only fix parameter $\theta_{3}, \theta_{4}$ and the impulsive matrix $W_{k}$, and then let $\theta_{5} P \leq Q_{1}, \theta_{6} P \geq Q_{2}$. Firstly, $P, Q_{1}, Q_{2}$ can be obtained by Theorem 7 via MATLAB LMI toolbox. Finally, $\theta_{5}, \theta_{6}$ can be calculated by following algebraic equations:

$$
\theta_{5}=\sqrt[n]{\frac{\operatorname{det}\left(Q_{1}\right)}{\operatorname{det}(P)}}, \quad \theta_{6}=\sqrt[n]{\frac{\operatorname{det}\left(Q_{2}\right)}{\operatorname{det}(P)}},
$$

or linear matrix inequalities:

$$
\theta_{5} P<Q_{1}, \quad \theta_{6} P>Q_{2}
$$

In $[22,25]$, the parameters $\beta_{4}$ and $r$ which are corresponding to parameter $\theta_{6}$ had been selected subjectively, which may cause result to lack fidelity. In addition, parameters $\theta_{3}, \theta_{4}$ are selectable variables which can increase the flexibility of the possible outcomes. Therefore, our results is more objective in some situations.

From the control point of view, in order to obtain the synchronization between the drive system (1) and the controlled response system (5), we design $\left(t_{k}, W_{k}\right), k \in \mathbb{N}$ as the impulsive controller [32]. Let $W_{k}=b I$ in Theorem 7, the following corollary holds.

Corollary 9. Assume that assumption (H1) holds. If there exist three $n \times n$ symmetric and positive definite matrices $P, Q_{1}^{\prime}, Q_{2}$, 


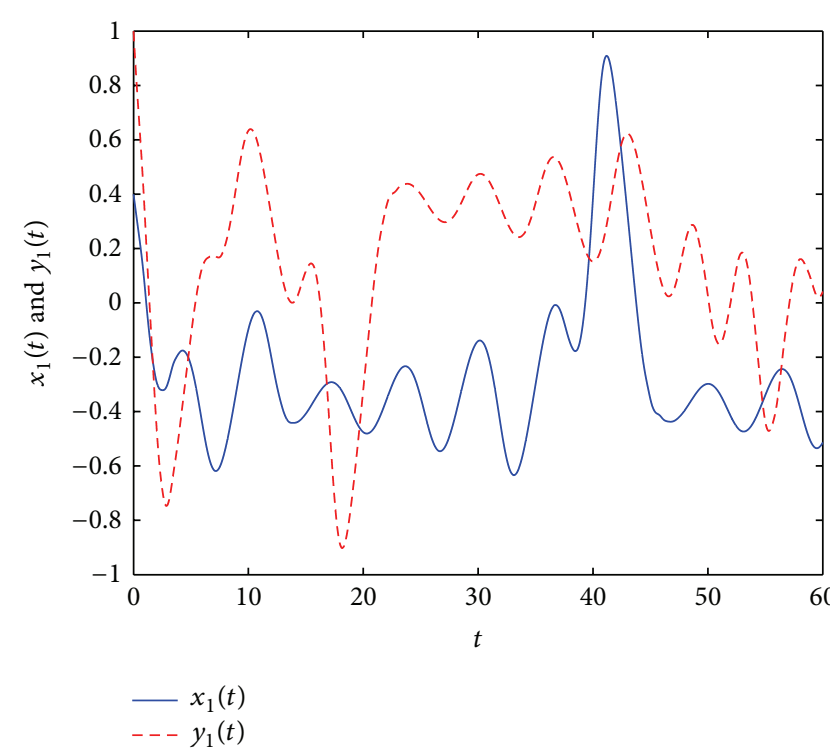

(a)

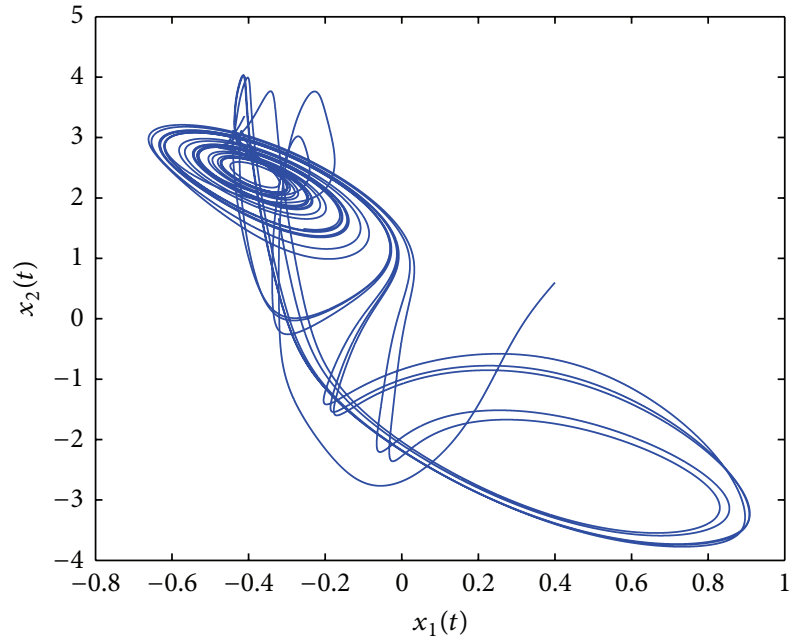

(c)

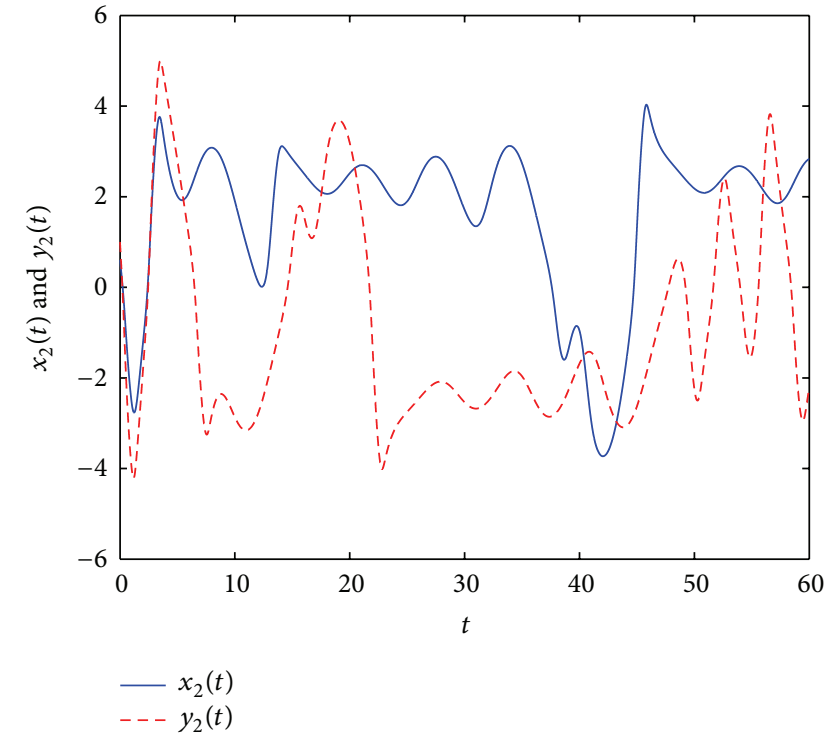

(b)

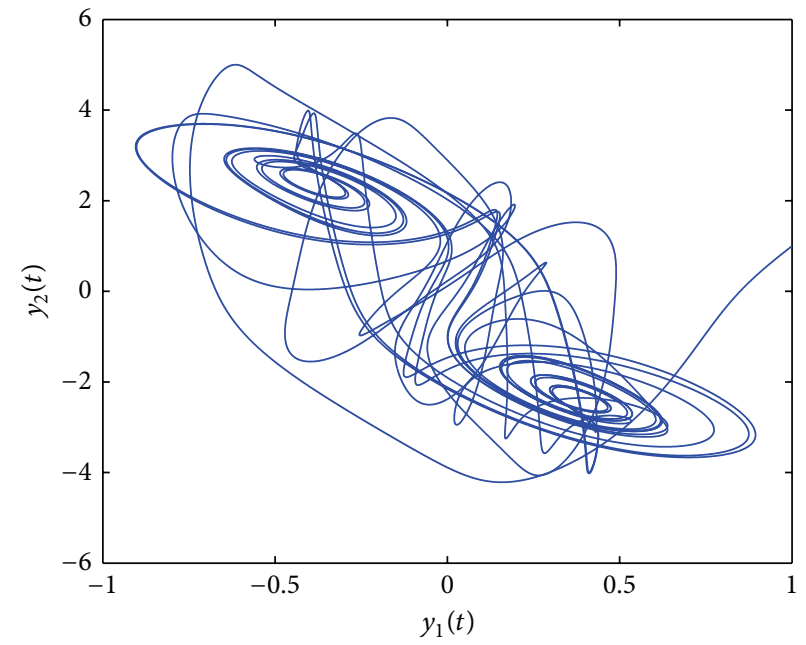

(d)

FIGURE 1: Time response of state variables and the phase plots of the drive system and response system in Example 12 without impulsive control.

seven constants $-2<b<0(b \neq-1), \theta_{1}, \theta_{2}, \theta_{5}, \theta_{6}>0,0<$ $\theta_{3}, \theta_{4}<2$ such that the following inequalities hold:

$$
\begin{gathered}
\Gamma \leq 0, \\
\sup \left\{t_{k}-t_{k-1}\right\}<-\frac{2 \ln |1+b|}{\theta_{6}}, \quad k \in \mathbb{N},
\end{gathered}
$$

where

$$
\Gamma=\left[\begin{array}{cccccc}
\Omega_{1}^{\prime} & P B & P C & 0 & 0 & 0 \\
\star & -\theta_{1} I & 0 & 0 & 0 & 0 \\
\star & \star & -\theta_{2} I & 0 & 0 & 0 \\
\star & \star & \star & \Omega_{2}-\theta_{5} P & 0 & 0 \\
\star & \star & \star & \star & \theta_{5} P-Q_{1} & 0 \\
\star & \star & \star & \star & \star & Q_{2}-\theta_{6} P
\end{array}\right]
$$

$$
\begin{aligned}
\Omega_{1}^{\prime}= & -A^{T} P-P A+\frac{\theta_{1}}{2 \theta_{3}-\theta_{3}^{2}} L_{1} L_{1}^{T}+\frac{Q_{1}}{(1+b)^{2}} \\
& -Q_{2}-\frac{2 \theta_{1}}{2-\theta_{3}} L_{2}, \\
& \Omega_{2}=\frac{\theta_{2}}{2 \theta_{4}-\theta_{4}^{2}} L_{1} L_{1}^{T}-\frac{2 \theta_{2}}{2-\theta_{4}} L_{2},
\end{aligned}
$$

then the systems (1) and (5) are asymptotically synchronized.

In particular, when $l_{i}^{-}=-l_{i}^{+}<0$ in (H1), the following corollary holds.

Corollary 10. Consider system (6) satisfying assumption (H1) with $l_{i}^{-}=-l_{i}^{+}<0$. If there exist three $n \times n$ symmetric and positive definite matrices $P, Q_{1}, Q_{2}$, seven constants 


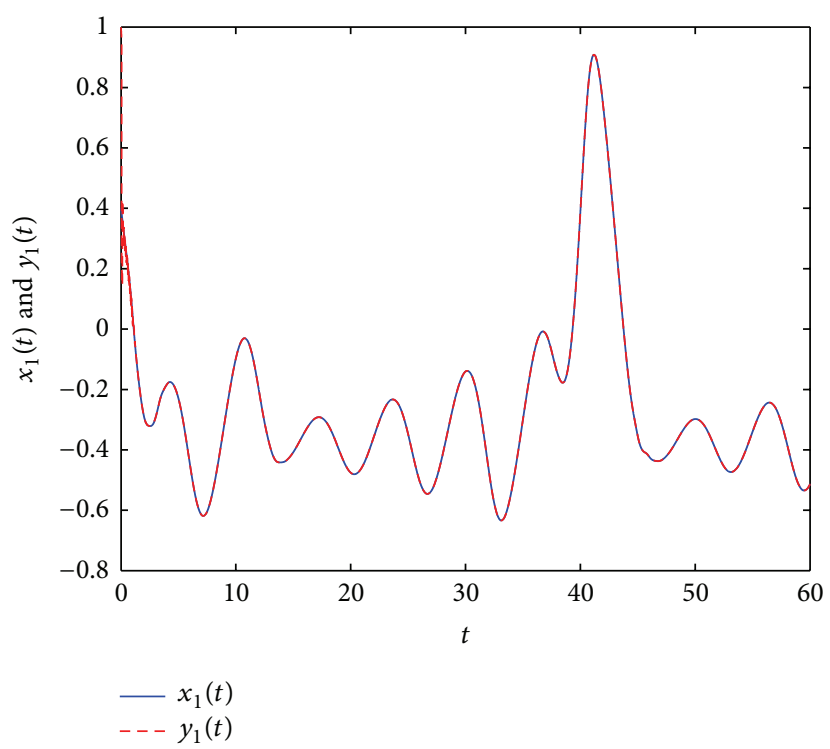

(a)

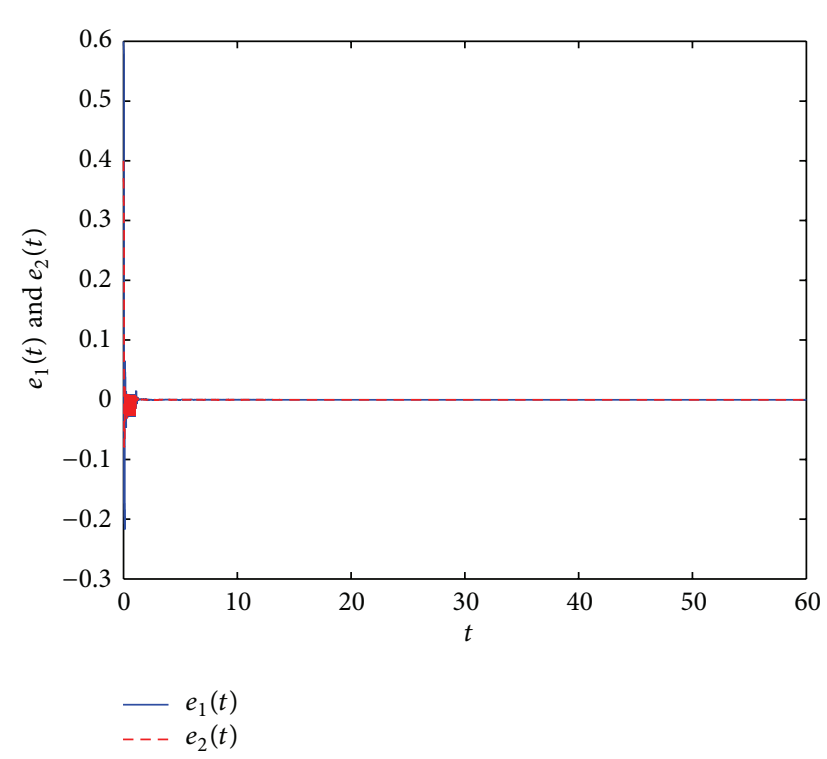

(c)

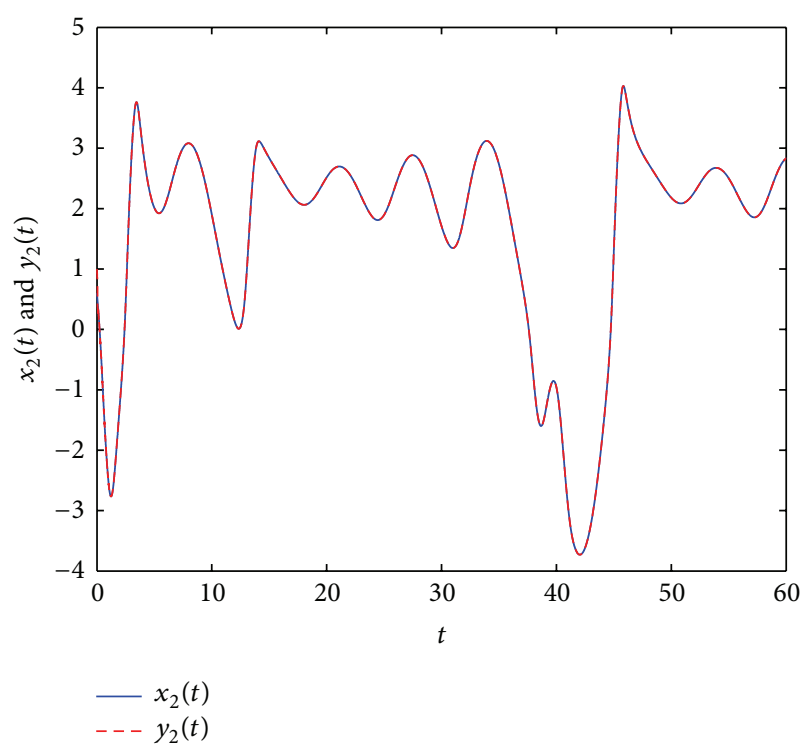

(b)

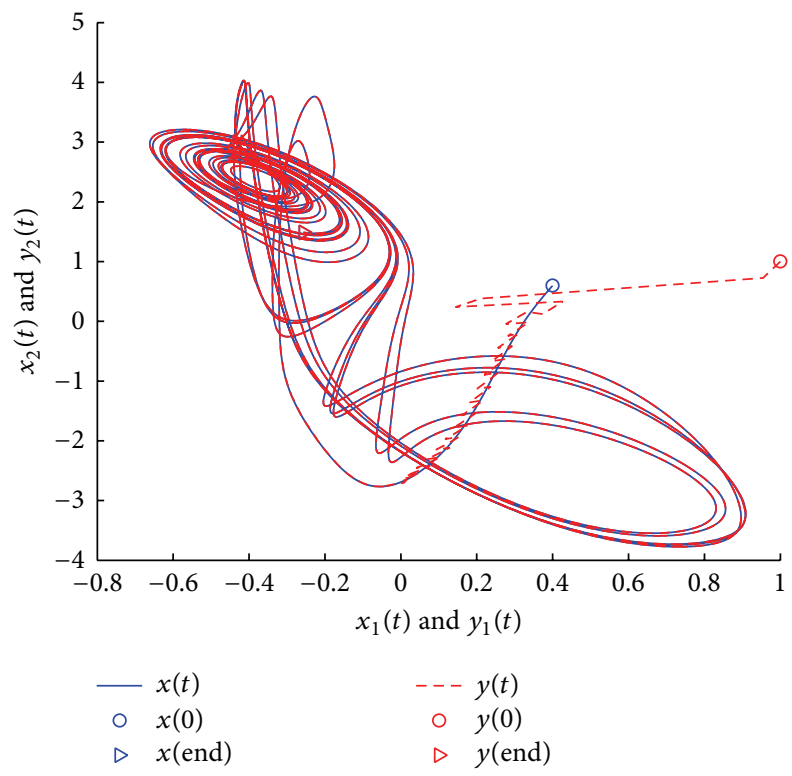

(d)

Figure 2: Time response of state variables, the synchronization errors, and the phase plots of the drive system and response system in Example 12 under the impulsive controller $(0.06 k,-1.3 I)$.

$-2<b<0(b \neq-1), \theta_{1}, \theta_{2}, \theta_{5}, \theta_{6}>0,0<\theta_{3}, \theta_{4}<2$ such that the following inequalities hold:

$$
\begin{gathered}
\Gamma \leq 0, \\
\sup \left\{t_{k}-t_{k-1}\right\}<-\frac{2 \ln |1+b|}{\theta_{6}}, \quad k \in \mathbb{N},
\end{gathered}
$$

where

$$
\Gamma=\left[\begin{array}{cccccc}
\Omega_{1}^{\prime \prime} & P B & P C & 0 & 0 & 0 \\
\star & -\theta_{1} I & 0 & 0 & 0 & 0 \\
\star & \star & -\theta_{2} I & 0 & 0 & 0 \\
\star & \star & \star & \Omega_{2}^{\prime}-\theta_{5} P & 0 & 0 \\
\star & \star & \star & \star & \theta_{5} P-Q_{1} & 0 \\
\star & \star & \star & \star & \star & Q_{2}-\theta_{6} P
\end{array}\right]
$$

$$
\begin{gathered}
\Omega_{1}^{\prime \prime}=-A^{T} P-P A+\frac{Q_{1}}{(1+b)^{2}}-Q_{2}-\frac{2 \theta_{1}}{2-\theta_{3}} L_{2}, \\
\Omega_{2}^{\prime}=-\frac{2 \theta_{2}}{2-\theta_{4}} L_{2},
\end{gathered}
$$

then the systems (1) and (5) are asymptotically synchronized.

Remark 11. The sufficient conditions in Theorem 7 and Corollaries 9 and 10 are all independent of the delay parameter but rely on the maximum impulsive interval $\sup \left\{t_{k}-t_{k-1}\right\}$ and the impulsive matrix $W_{k}$, which plays a fundamental role when the size of the delay is unknown. 


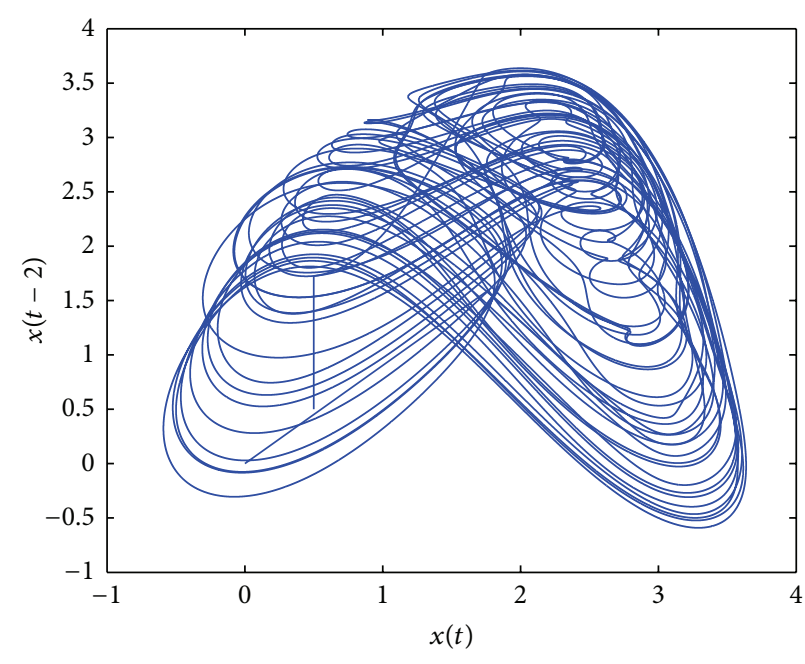

(a)

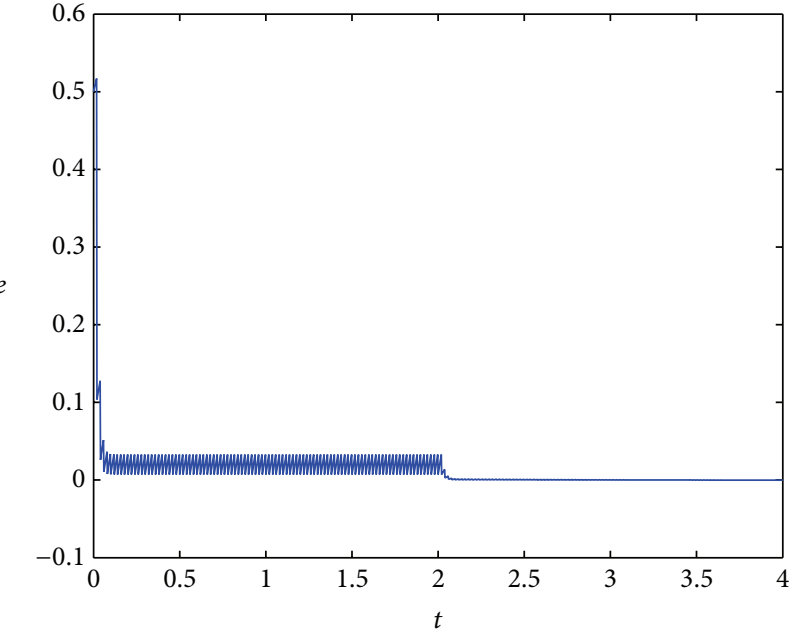

(b)

FIGURE 3: Chaotic attractor of Ikeda-type neural network in Example 14 and the synchronization error of the drive system and response system in Example 14.

TABLE 1: When the impulsive matrix $W_{k}$ is given, $\sup _{k}\left\{t_{k}-t_{k-1}\right\}, k \in \mathbb{N}$ corresponding to different $\left(\theta_{3}, \theta_{4}\right)$ and algorithms.

\begin{tabular}{|c|c|c|c|c|c|}
\hline \multirow{2}{*}{$\left(W_{k}\right.$, Algorithms $)$} & \multicolumn{5}{|c|}{$\left(\theta_{3}, \theta_{4}\right)$} \\
\hline & $(0.1,0.1)$ & $(0.3,0.3)$ & $(0.5,0.5)$ & $(0.7,0.7)$ & $(0.9,0.9)$ \\
\hline$\left(W_{k}=-1.1 I,(31)\right)$ & 0.0202 & 0.0288 & 0.0305 & 0.0356 & 0.0369 \\
\hline$\left(W_{k}=-1.1 I,(32)\right)$ & $2.8349 e-4$ & $1.0980 e-4$ & $5.3202 e-4$ & $7.2925 e-4$ & $8.9236 e-4$ \\
\hline$\left(W_{k}=-1.3 I,(31)\right)$ & 0.0310 & 0.0401 & 0.0490 & 0.0718 & 0.0701 \\
\hline$\left(W_{k}=-1.3 I,(32)\right)$ & 0.0025 & 0.0163 & 0.0200 & 0.0259 & 0.0259 \\
\hline$\left(W_{k}=-1.5 I,(31)\right)$ & 0.0175 & 0.0266 & 0.0335 & 0.0483 & 0.0516 \\
\hline$\left(W_{k}=-1.5 I,(32)\right)$ & 0.0058 & 0.0094 & 0.0122 & 0.0023 & 0.0022 \\
\hline$\left(W_{k}=-1.7 I,(31)\right)$ & 0.0088 & 0.0168 & 0.0213 & 0.0245 & 0.0261 \\
\hline$\left(W_{k}=-1.7 I,(32)\right)$ & 0.0027 & 0.0020 & $9.4549 e-04$ & 0.0056 & 0.0059 \\
\hline$\left(W_{k}=-1.9 I,(31)\right)$ & 0.0029 & 0.0057 & 0.0074 & 0.0093 & 0.0089 \\
\hline$\left(W_{k}=-1.9 I,(32)\right)$ & $2.7931 e-04$ & 0.0011 & 0.0015 & 0.0019 & 0.0016 \\
\hline
\end{tabular}

\section{Numerical Results}

In order to illustrate the feasibility of our above-established criteria in the preceding sections, we provide two concrete examples. Throughout the simulations, we use the IMEX implicit Euler method.

Example 12. Consider a two-dimensional chaotic delayed neural networks as the drive system (1), where the initial condition $\phi(s)=(0.6,0.4)^{T}, s \in[-\tau, 0], f(x)=\tanh (x), \tau(t)=$ $1, J=(0,0)^{T}$; then $L_{1}=(1,1)^{T}, L_{2}=(0,0)^{T}$, and the parameter matrices $A, B, C$ are given as follows:

$$
\begin{gathered}
A=\left(\begin{array}{ll}
1 & 0 \\
0 & 1
\end{array}\right), \quad B=\left(\begin{array}{cc}
2.0 & -0.1 \\
-5.0 & 3
\end{array}\right), \\
C=\left(\begin{array}{ll}
-1.5 & -0.1 \\
-0.2 & -2.5
\end{array}\right) .
\end{gathered}
$$

The corresponding response system is designed as (5), where the initial condition $\varphi(s)=(1,1)^{T}, s \in[-\tau, 0]$. From [33], we know that the system (1) of Example 12 has a chaotic attractor which can be seen from Figure 1. Figures 1(a)-1(d) show the time response of state variables and the phase plots of the drive system and response system in Example 12 without impulsive control.

By choosing $\theta_{3}=\theta_{4}=0.7, b=-1.3$ and then using MATLAB LMI toolbox and (31), we can obtain the following feasible solutions to LMIs and $\theta_{5}, \theta_{6}$ in Corollary 9:

$$
\begin{gathered}
P=\left(\begin{array}{ll}
0.0339 & 0.0022 \\
0.0022 & 0.0111
\end{array}\right), \quad Q_{1}=\left(\begin{array}{cc}
0.0255 & -0.0004 \\
-0.0004 & 0.0266
\end{array}\right), \\
Q_{2}=\left(\begin{array}{cc}
0.6427 & -0.0023 \\
-0.0023 & 0.6488
\end{array}\right), \\
\theta_{1}=0.2070, \quad \theta_{2}=0.0175, \quad \theta_{5}=1.3526, \\
\theta_{6}=33.5145 .
\end{gathered}
$$

Thus, from Corollary 9 we can calculate that the maximum impulsive interval satisfies $\sup \left\{t_{k}-t_{k-1}\right\}<0.0718$. 
With the same initial conditions as given above and $t_{k}-$ $t_{k-1}=0.06$, Figures $2(\mathrm{a})-2(\mathrm{~d})$ depict the time response of state variables, the synchronization errors, and the phase plots of the drive system and response system in Example 12 under the impulsive controller $(0.06 k,-1.3 I)$.

Remark 13. In Table 1, we list $\sup _{k}\left\{t_{k}-t_{k-1}\right\}$ corresponding to different $\left(\theta_{3}, \theta_{4}\right)$ and algorithms when the impulsive matrix $W_{k}$ is given. For each $\left(\theta_{3}, \theta_{4}\right)$, the $\left(\theta_{6}, \sup _{k}\left\{t_{k}-t_{k-1}\right\}\right)$ is obtained by solving the LMIs in Corollary 9 and (31) or (32). From the results in Table 1, we can see that the obtained maximum stable impulsive interval $\sup _{k}\left\{t_{k}-t_{k-1}\right\}$ by using (31) is better than (32) when the impulsive matrix $W_{k}$ is given. And when $\theta_{3}, \theta_{4}$ are chosen in interval $[1,2)$, we will have the same results which are chosen as $2-\theta_{3}, 2-\theta_{4}$.

Example 14. Consider the Ikeda-type neural network [34] as the drive system (1); when $A=1.0, B=0, C=4.0, f(x)=$ $\sin (x), \tau(t)=2$, the system (1) exhibits chaotic behavior (see Figure 3(a)). And the initial condition $\phi(s)=0.5, s \in[-\tau, 0]$. It is easy to obtain that $L_{1}=0, L_{2}=-1$.

The corresponding response system is designed as (5), where the initial condition $\varphi(s)=1, s \in[-\tau, 0]$.

By choosing $\theta_{3}=\theta_{4}=0.5, b=-0.8$ and then using MATLAB LMI toolbox and (31), we can obtain the following feasible solutions to LMIs and $\theta_{5}, \theta_{6}$ in Corollary 10:

$$
\begin{gathered}
P=0.0068, \quad Q_{1}=0.0161, \quad Q_{2}=0.8861, \\
\theta_{1}=0.2283, \quad \theta_{2}=0.0083, \quad \theta_{5}=2.3538, \quad \theta_{6}=129.6849 .
\end{gathered}
$$

Hence, the designed impulsive controller is

$$
W_{k}=-0.8, \quad \sup \left\{t_{k}-t_{k-1}\right\}<0.0248 .
$$

With the same initial conditions as given above, the simulations of the synchronization error in Example 14 under the impulsive controller $(0.02 k,-0.8)$ are shown in Figure 3(b).

\section{Conclusion}

In this paper, the impulsive synchronization problem of chaotic delayed neural networks has been investigated. Some new criterions which ensure that the coupled chaotic delayed neural networks can be asymptotically synchronized have been derived in terms of linear matrix inequalities (LMIs) by using Lyapunov stability theorem, impulsive control theory, and linear matrix inequality (LMI) technique. The desired impulsive controller which is with respect to the stable impulsive interval and the impulsive matrix is established, its existence can be verified effectively by the simulations. It is worthwhile to mention that the positive constants set $\left(\theta_{3}, \theta_{4}\right)$ can increase the flexibility for the design of the impulsive controller. Moreover, the stable impulsive interval can be calculated combining MATLAB LMI toolbox and one of the two given equations objectively.

Finally, two illustrative examples are given to show the applicability and usefulness of the proposed results.

\section{Conflict of Interests}

The authors declare that there is no conflict of interests regarding the publication of this paper.

\section{Acknowledgment}

The authors would like to thank the editor and the anonymous reviewers for their careful comments and suggestions to improve the quality of the paper. This work is jointly supported by Opening Fund of Artificial Intelligence Key Laboratory of Sichuan Province under Grant no. 2011RK01, Opening Fund of Geomathmathematics Key Laboratory of Sichuan Province under Grant no. scsxdz2011010, the Scientific Research Fund of Sichuan University of Science and Engineering under Grant no. 2011PY08, and the Graduate Innovation Fund of Sichuan University of Science and Engineering under Grant no. y2013021.

\section{References}

[1] S. Mohamad, K. Gopalsamy, and H. Akça, "Exponential stability of artificial neural networks with distributed delays and large impulses," Nonlinear Analysis. Real World Applications, vol. 9, no. 3, pp. 872-888, 2008.

[2] X. Li, "Global robust stability for stochastic interval neural networks with continuously distributed delays of neutral type," Applied Mathematics and Computation, vol. 215, no. 12, pp. 4370-4384, 2010.

[3] R. Rakkiyappan and P. Balasubramaniam, "Dynamic analysis of Markovian jumping impulsive stochastic Cohen-Grossberg neural networks with discrete interval and distributed timevarying delays," Nonlinear Analysis. Hybrid Systems, vol. 3, no. 4, pp. 408-417, 2009.

[4] K. Gopalsamy, "Leakage delays in BAM," Journal of Mathematical Analysis and Applications, vol. 325, no. 2, pp. 1117-1132, 2007.

[5] X. Li and Z. Chen, "Stability properties for Hopfield neural networks with delays and impulsive perturbations," Nonlinear Analysis. Real World Applications, vol. 10, no. 5, pp. 3253-3265, 2009.

[6] J. H. Park and O. M. Kwon, "Global stability for neural networks of neutral-type with interval time-varying delays," Chaos, Solitons and Fractals, vol. 41, no. 3, pp. 1174-1181, 2009.

[7] S. K. Han, C. Kurrer, and Y. Kuramoto, "Dephasing and bursting in coupled neural oscillators," Physical Review Letters, vol. 75, no. 17, pp. 3190-3193, 1995.

[8] T. Kwok and K. A. Smith, "Experimental analysis of chaotic neural network models for combinatorial optimization under a unifying framework," Neural Networks, vol. 13, no. 7, pp. 731$744,2000$.

[9] Z. Tan and M. K. Ali, "Associative memory using synchronization in a chaotic neural network," International Journal of Modern Physics C, vol. 12, no. 1, pp. 19-29, 2001.

[10] K. Aihara, T. Takabe, and M. Toyoda, "Chaotic neural networks," Physics Letters A, vol. 144, no. 6-7, pp. 333-340, 1990.

[11] V. Milanovic and M. E. Za-ghloul, "Synchronization of chaotic neural networks and applications to communications," International Journal of Bifurcation and Chaos, vol. 612, pp. 2571-2585, 1996.

[12] T. Yang and L. O. Chua, "Impulsive stabilization for control and synchronization of chaotic systems: theory and application 
to secure communication," IEEE Transactions on Circuits and Systems. I. Fundamental Theory and Applications, vol. 44, no. 10, pp. 976-988, 1997.

[13] C. Li, X. Liao, and K.-w. Wong, "Chaotic lag synchronization of coupled time-delayed systems and its applications in secure communication," Physica D, vol. 194, no. 3-4, pp. 187-202, 2004.

[14] C.-J. Cheng and C.-B. Cheng, "An asymmetric image cryptosystem based on the adaptive synchronization of an uncertain unified chaotic system and a cellular neural network," Communications in Nonlinear Science and Numerical Simulation, vol. 18, no. 10, pp. 2825-2837, 2013.

[15] V. Perez-Munuzuri, V. Perez-Villar, and L. O. Chua, "Autowaves for image processing on a two-dimensional CNN array of excitable nonlinear circuits: flat and wrinkled labyrinths," IEEE Transactions on Circuits and Systems I, vol. 40, no. 3, pp. 174-181, 1993.

[16] J. J. Fox, C. Jayaprakash, D. Wang, and S. R. Campbell, "Synchronization in relaxation oscillator networks with conduction delays," Neural Computing, vol. 13, no. 5, pp. 1003-1021, 2001.

[17] Q. Gan, "Adaptive synchronization of Cohen-Grossberg neural networks with unknown parameters and mixed time-varying delays," Communications in Nonlinear Science and Numerical Simulation, vol. 17, no. 7, pp. 3040-3049, 2012.

[18] V. I. Utkin, Sliding Modes in Control and Optimization, Springer, Berlin, Germany, 2001.

[19] J. Heagy, T. Carroll, and L. Pecora, "Experimental and numerical evidence for riddled basins in coupled chaotic systems," Physical Review Letters, vol. 73, no. 26, pp. 3528-3531, 1994.

[20] J. H. Park, "Synchronization of cellular neural networks of neutral type via dynamic feedback controller," Chaos, Solitons and Fractals, vol. 42, no. 3, pp. 1299-1304, 2009.

[21] H. Zhao and Q. Zhang, "Global impulsive exponential antisynchronization of delayed chaotic neural networks," Neurocomputing, vol. 74, no. 4, pp. 563-567, 2011.

[22] X. Li and R. Rakkiyappan, "Impulse controller design for exponential synchronization of chaotic neural networks with mixed delays," Communications in Nonlinear Science and Numerical Simulation, vol. 18, no. 6, pp. 1515-1523, 2013.

[23] P. Li, J. Cao, and Z. Wang, "Robust impulsive synchronization of coupled delayed neural networks with uncertainties," Physica A, vol. 373, pp. 261-272, 2007.

[24] Y. Zhang and J. Sun, "Robust synchronization of coupled delayed neural networks under general impulsive control," Chaos, Solitons \& Fractals, vol. 41, no. 3, pp. 1476-1480, 2009.

[25] C. Li, X. Liao, X. Yang, and T. Huang, "Impulsive stabilization and synchronization of a class of chaotic delay systems," Chaos, vol. 15, no. 4, Article ID 043103, 2005.

[26] H. K. Khalil, Nonlinear Systems, Prentice Hall, Upper Saddle River, NJ, USA, 1996.

[27] T. Yang, Impulsive Control Theory, Springer, Berlin, Germany, 2001.

[28] J. Yan and J. Shen, "Impulsive stabilization of functional-differential equations by Lyapunov-Razumikhin functions," Nonlinear Analysis. Theory, Methods \& Applications, vol. 37, no. 2, pp. 245-255, 1999.

[29] A. Berman and R. J. Plemmons, Nonnegative Matrices in the Mathematical Sciences, Society for Industrial and Applied Mathematics (SIAM), Philadelphia, Pa, USA, 1994.

[30] Y. Y. Wang, L. Xie, and C. E. de Souza, "Robust control of a class of uncertain nonlinear systems," Systems \& Control Letters, vol. 19, no. 2, pp. 139-149, 1992.
[31] S. Boyd, L. El Ghaoui, E. Feron, and V. Balakrishnan, Linear Matrix Inequalities in System and Control Theory, Society for Industrial and Applied Mathematics (SIAM), Philadelphia, Pa, USA, 1994

[32] Z. Yang and D. Xu, "Stability analysis and design of impulsive control systems with time delay," IEEE Transactions on Automatic Control, vol. 52, no. 8, pp. 1448-1454, 2007.

[33] H. T. Lu, "Chaotic attractors in delayed neural networks," Physics Letters A, vol. 298, no. 2-3, pp. 109-116, 2002.

[34] K. Ikeda, "Multiple-valued stationary state and its instability of the transmitted light by a ring cavity system," Optics Communications, vol. 30, no. 2, pp. 257-261, 1979. 


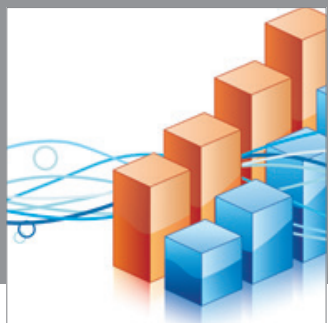

Advances in

Operations Research

mansans

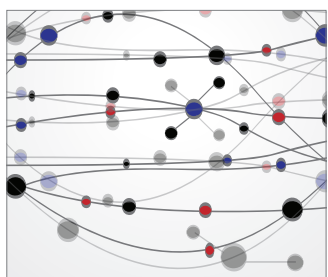

The Scientific World Journal
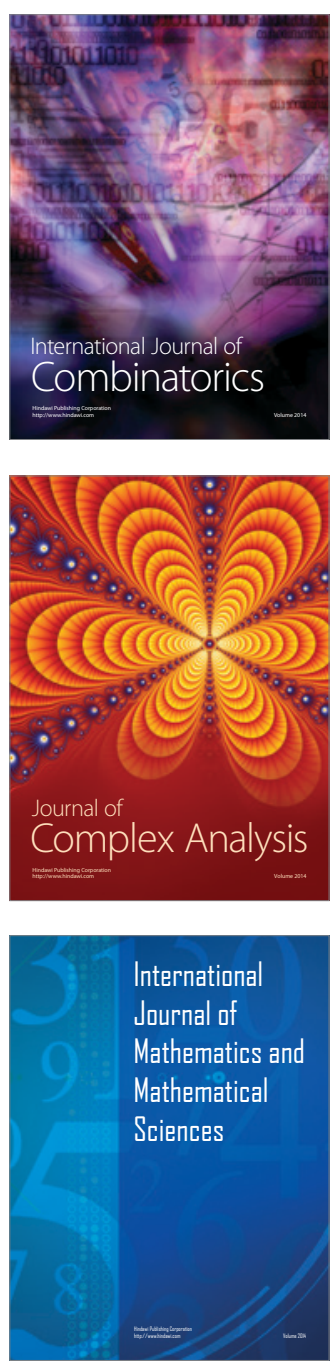
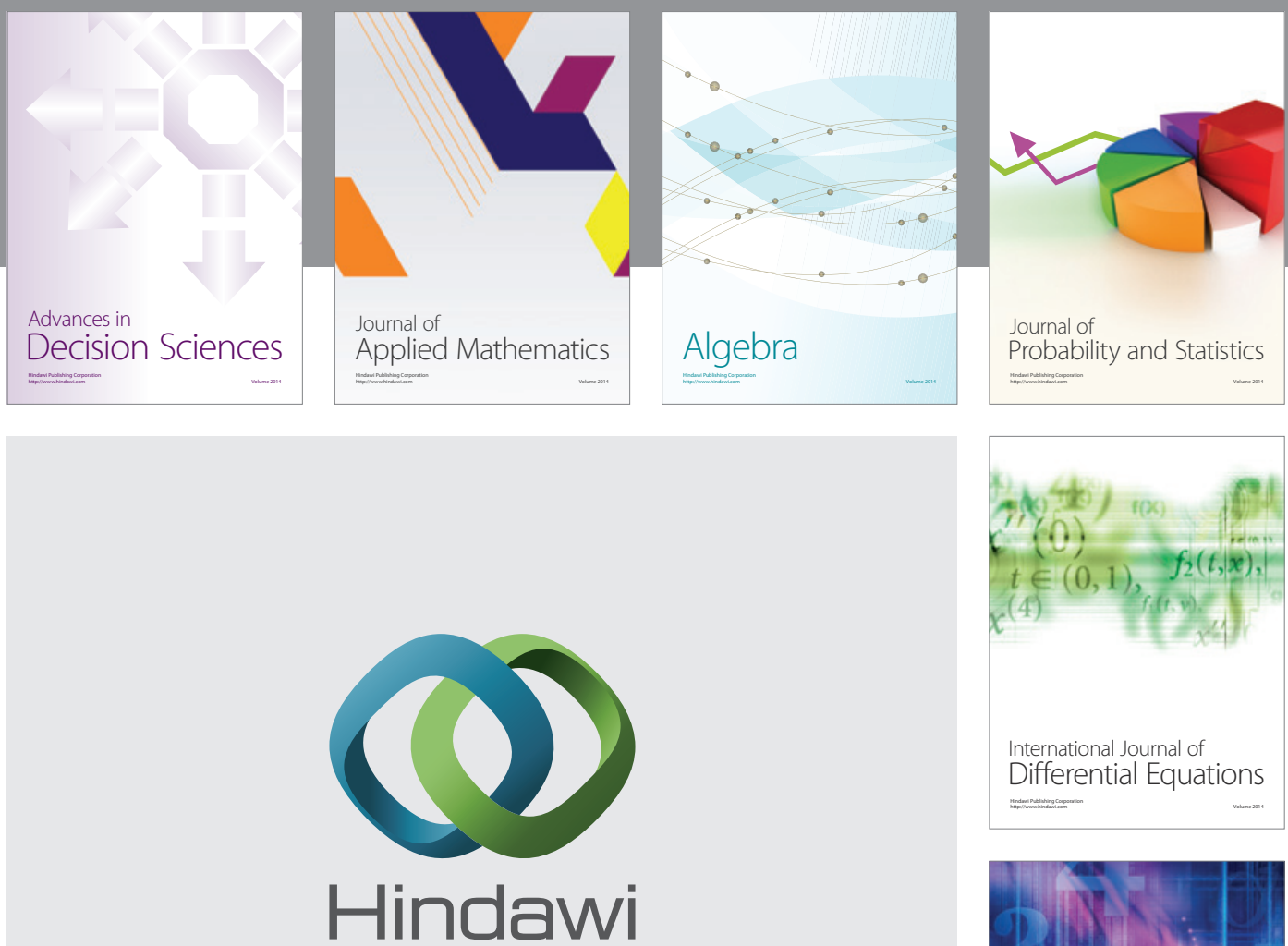

Submit your manuscripts at http://www.hindawi.com
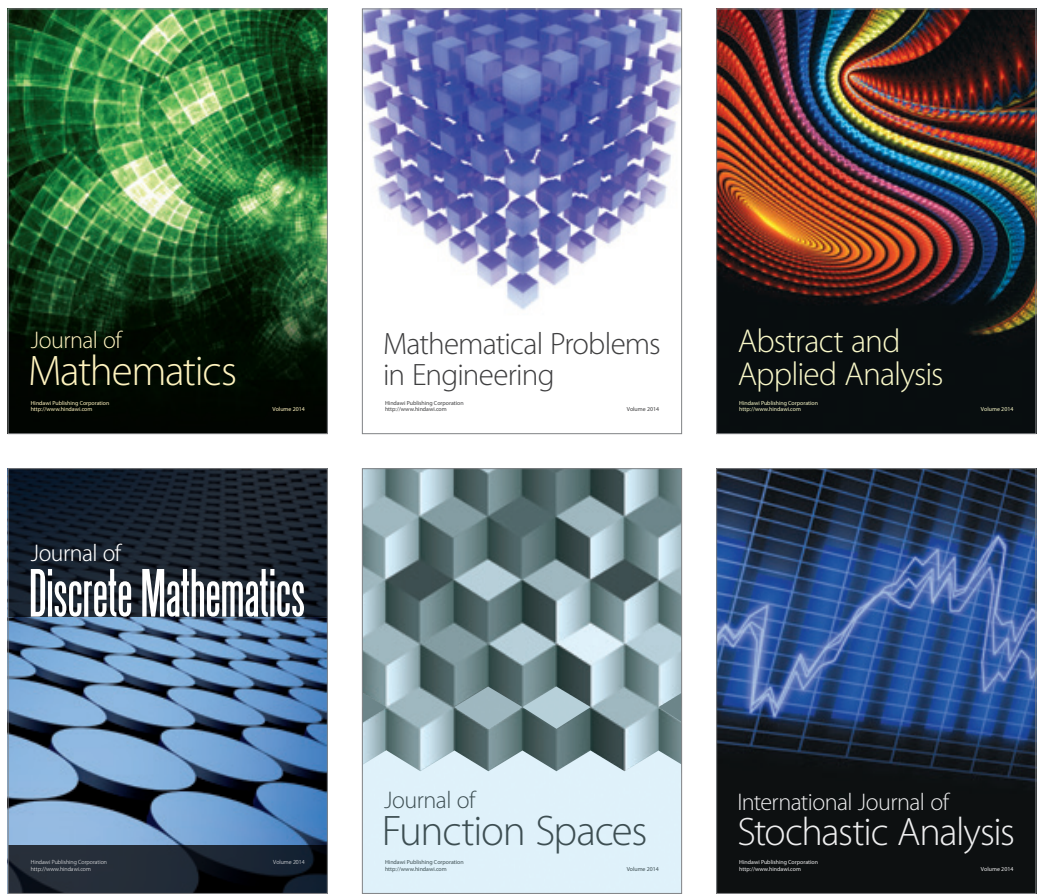

Journal of

Function Spaces

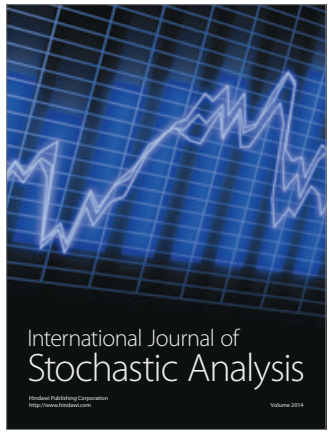

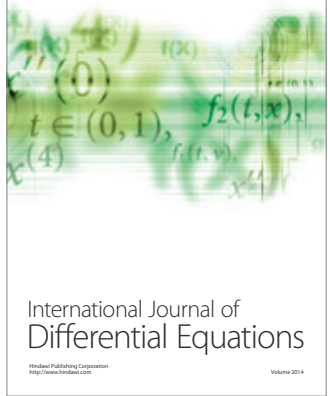
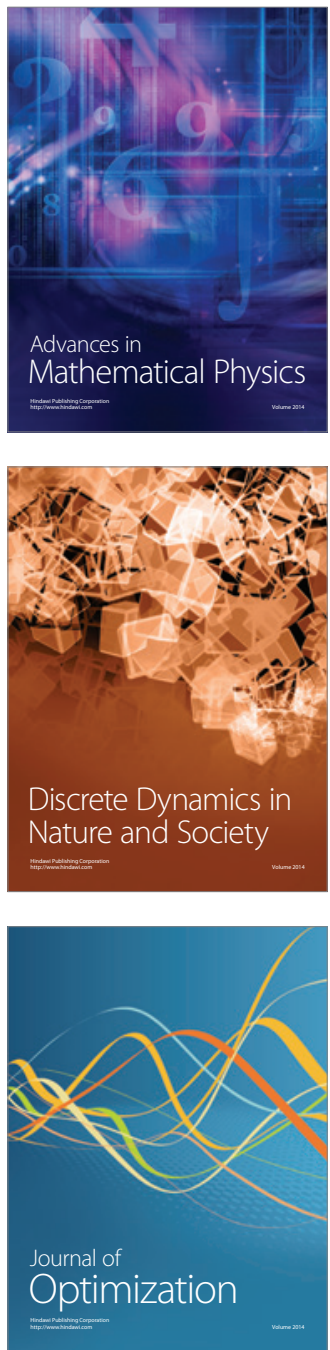